\title{
Energetics of (dis)assembly of the ternary SNARE complex
}

\author{
Wei Liu ${ }^{1,2 *}$ and Vladimir Parpura ${ }^{1,2 *}$ \\ Atomic Force Microscopy and Nanotechnology Laboratories, Department of Neurobiology, Center for Glial Biology in Medicine, Civitan International Research Center, \\ Birmingham, AL, USA \\ 2 Evelyn F. McKnight Brain Institute, University of Alabama, Birmingham, AL, USA \\ *Correspondence: liuw@uab.edu; vlad@uab.edu
}

\section{A commentary on}

Is assembly of the SNARE complex enough to fuel membrane fusion?

by Wiederhold K., and Fasshauer, D. (2009). J. Biol. Chem. 284, 13143-13152.

The soluble $N$-ethylmaleimide-sensitive fusion protein attachment protein receptor (SNARE) complex (Sollner et al., 1993) plays a central role in the process of exocytosis whereby vesicles fuse with the plasma membrane to release their cargo of transmitter molecules into the extracellular space. In the majority of neurons, this complex is composed of the vesicular protein synaptobrevin $2(\mathrm{Sb} 2)$, and two proteins located at the plasma membrane, syntaxin $(\mathrm{Sx})$ and synaptosome-associated protein of $25 \mathrm{kDa}$ (SNAP25). The energetics of (dis)assembly of the ternary SNARE complex is critical for understanding of exocytosis, in particular to their role in mediating vesicular fusions to and/or pinching off the plasma membrane.

The energy required for disassembly of the ternary SNARE complex has been recently assessed by two different groups (Li et al., 2007; Liu et al., 2009). In both studies SNARE proteins were immobilized to surfaces. One surface contained a $\mathrm{Sx}$ SNAP25 binary complex, while the other $\mathrm{Sb} 2$. These surfaces were brought into contact allowing for the formation of the ternary complex, before the surfaces were pulled apart to dismantle the complex. Using surface force apparatus (SFA), Li et al. (2007) revealed a change in free, presumably Gibbs $(\Delta G)$, energy of $21 \mathrm{kcal} \mathrm{mol}^{-1}\left(35 k_{B} T\right)$ assigned to a disassembly of single SNARE complex. Liu et al. (2009) using Atomic Force Microscopy (AFM) in force spectroscopy mode reported the enthalpic changes $(\Delta H)$ of $25.7 \mathrm{kcal} \mathrm{mol}^{-1}\left(43 k_{B} T\right)$, as well changes in free energy $(\Delta G)$ of $13.8-18.0 \mathrm{kcal} \mathrm{mol}^{-1}$ (23-30 $\left.k_{B} T\right)$ and entropy $(-T \Delta S)$ for a disassembly of single ternary SNARE complex (Table 1). Both SFA and AFM approaches, however, could not be used to measure the energetics of the assembly of the complex.

Wiederhold and Fasshauer (2009) investigated the ternary SNARE complex assembly by isothermal titration calorimetry (ITC). Various combinations of SNARE proteins were put in a thermally insulated cell and syringe, and then were mixed by injection from the syringe to the cell, while measuring the thermodynamic properties. To avoid formation of the Sx1-SNAP25 binary complex with 2:1 stoichiometry, referred to as a "dead-end species" (Weninger et al., 2008) since it does not represent a reactive Sb2 binding site (Pobbati et al., 2006), SNAP25A was injected into a mixture of Sx1A (H3 domain) and Sb2 [cytosolic domain; amino acids (aa) 1-96] to form the ternary SNARE complex. In these conditions there was extremely large favorable $\Delta H$ of $-112.8 \mathrm{kcal} \mathrm{mol}^{-1}$ recorded with the positive entropy changes $\left(102.4 \mathrm{kcal} \mathrm{mol}^{-1}\right)$, reflecting the major conformation change during complex assembly, and resulting in $\Delta G$ of $-10.4 \mathrm{kcal} \mathrm{mol}^{-1}\left(-17.4 k_{B} T\right)$ (Table 1).

The ITC measurements above represent energetics of a non-sequential ternary SNARE complex formation, rather than the sequential interactions in which Sb2 binds to a preformed Sx1-SNAP25 binary complex with 1:1 stoichiometry. To addrsess this issue the authors cleverly designed experiments using so-called " $\Delta N$ complex"(Pobbati et al., 2006). Here, the 1:1 Sx-SNAP25 binary complex can be stabilized by addition of C-terminal fragment of Sb2 SNARE domain (aa 49-96), and then purified. $\Delta N$ complex was titrated by injection of the entire cytosolic domain of $\mathrm{Sb} 2_{1-96}$, which binds to the complex and displaces $\mathrm{Sb} 2_{49-96}$ as confirmed by

\begin{tabular}{|c|c|c|c|c|c|c|c|}
\hline \multirow[t]{2}{*}{ Disassembly } & \multirow[t]{2}{*}{ Surface 1} & \multirow[t]{2}{*}{ Surface 2} & $\Delta \mathrm{H}$ & \multicolumn{2}{|c|}{$-\mathrm{T} \Delta \mathrm{S}$} & \multicolumn{2}{|c|}{$\Delta \mathrm{G}$} \\
\hline & & & $\mathrm{kcal} \mathrm{mol}^{-1}$ & \multicolumn{2}{|c|}{$\mathrm{kcal} \mathrm{mol}^{-1}$} & \multicolumn{2}{|c|}{ kcal mol-1 } \\
\hline \multirow[t]{2}{*}{ SFA } & \multirow[t]{2}{*}{$\mathrm{S} \times 1 \mathrm{~A}_{\mathrm{cd}}-\mathrm{SNAP} 25^{*}$} & \multirow[t]{2}{*}{$\mathrm{Sb} 2_{\mathrm{cd}}{ }^{*}$} & & & & \multicolumn{2}{|c|}{$21.0 \pm 4.2$} \\
\hline & & & & Eyring & Kramers & Eyring & Kramers \\
\hline AFM & $\mathrm{S} \times 1 \mathrm{~A}_{\mathrm{cd}}-\mathrm{SNAP} 25 \mathrm{~B}$ & $\mathrm{Sb} 2_{\mathrm{cd}}$ & $25.7 \pm 3.0$ & $-7.7 \pm 3.3$ & $-11.9 \pm 3.3$ & $18.0 \pm 4.5$ & $13.8 \pm 4.5$ \\
\hline \multirow[t]{3}{*}{ ITC } & $\mathrm{S} \times 1 \mathrm{AH} 3 / \mathrm{Sb} 2_{c d}$ & SNAP25A & $-112.8 \pm 2.5$ & \multicolumn{2}{|c|}{102.4} & \multicolumn{2}{|c|}{-10.4} \\
\hline & $\Delta \mathrm{N}$ complex & $\mathrm{Sb} 2_{\mathrm{cd}}$ & $-29.9 \pm 0.3$ & \multicolumn{2}{|c|}{18.1} & \multicolumn{2}{|c|}{-11.8} \\
\hline & $\Delta \mathrm{N}$ complex & $\mathrm{Sb} 2_{1-52}$ & $-22.7 \pm 0.2$ & \multicolumn{2}{|c|}{12.1} & \multicolumn{2}{|c|}{-10.6} \\
\hline
\end{tabular}

To convert from kcal mol${ }^{1}$ to $\mathrm{k}_{B} \mathrm{~T}_{\text {multiply with 1.67. Sb2 }}$ cd corresponds to its 1-96 amino acids, Sb2 ${ }_{1-96}$. cd, cytosolic domain.

*Derived from mouse cDNA; all other protein sequences are based on rat cDNA. 
fluorescence anisotropy measurements in parallel experiments. There was a favorable $\Delta H$ of $-29.9 \mathrm{kcal} \mathrm{mol}^{-1}\left(-49.9 k_{B} T\right)$ with $\Delta G$ of $-11.8 \mathrm{kcal} \mathrm{mol}^{-1}\left(-19.7 k_{B} T\right)$, the former markedly reduced from, while the latter fairly similar to, measurements when SNAP25 was injected into Sx/Sb2 mixture (Table 1). One concern with this approach is how much of the observed energy is related to the binding of the entire cytosolic domain Sb2 ${ }_{1-96}$ to the complex as opposed to the displacement of the $\mathrm{Sb} 2_{49-96}$ fragment. The authors again used a clever approach by titrating the $\Delta N$ complex with a shorter N-terminal fragment of Sb2 SNARE domain $S b 2_{1-52}$ which binds to $\Delta N$ complex, but does not displace $\mathrm{Sb}{ }_{49-96}$ fragment, as confirmed by fluorescence anisotropy. Thus, two overlapping Sb2 fragments bind at the same time to a Sx-SNAP25 complex, forming a complex resembling the ternary SNARE complex. The ITC data were surprisingly similar to those obtained when a $\Delta N$ complex was titrated by injection of $\mathrm{Sb} 2_{1-96}$ implicating that binding of the Nterminal part of $\mathrm{Sb} 2$ contributes to most of energetics in Sb2 binding to the binary Sx-SNAP25 complex, while C-terminal Sb2 binding plays a lesser role, which was confimed in subsequent experiments using various fragments of Sb2. Taken together it appears that binding of Sb2 to a preformed Sx-SNAP25 heterodimer generates $\sim 20 k_{B} T$ of free energy as measured by ITC. Interestingly, this figure does not differ very much from the measurements of free energy for the disassembly of the ternary SNARE complex using AFM.

The reported amount of energy stored within a single ternary SNARE complex could be sufficient to cause hemifusion, since this process is estimated to require $\sim 13 k_{B} T$, while an energy barrier for full fusion is estimated to be $\sim 46 k_{B} T$ (Markin and Albanesi, 2002). Thus, if free energy generated from the formation of ternary SNARE complexes could be used for vesicular and plasma membranes fusion, this process would probably require several ternary SNARE complexes. Alternatively, if the energy released during the formation of complexes could not be applied towards vesicular fusions, but rather is simply consumed in the tethering/docking of vesicles at the plasma membrane, this would indicate that there should be an additional source of mechanical force/energy to drive vesicular fusions, perhaps from the combined effect of $\mathrm{Ca}^{2+}$-dependent synaptotagmin actions and its interactions with SNARE proteins. Of course, these two possibilities accounting for enthalpy/Gibbs free energy are not mutually exclusive.

\section{ACKNOWLEDGEMENTS}

We thank Randy F. Stout, Jr. and Axel T. Brunger for their comments on previous versions of this commentary. The authors' work is supported by a grant from the National Institute of Mental Health $(\mathrm{MH}$
069791 to VP). VP dedicates this work to Glenn I. Hatton, whose energy and creativity inspired new views of astrocyte-neuronal interactions.

\section{REFERENCES}

Li, F., Pincet, F., Perez, E., Eng, W. S., Melia, T. J., Rothman, J. E., and Tareste, D. (2007). Energetics and dynamics of snarepin folding across lipid bilayers. Nat. Struct. Mol. Biol. 14, 890-896.

Liu, W., Montana, V., Parpura, V., and Mohideen, U. (2009). Single molecule measurements of interaction free energies between the proteins within binary and ternary snare complexes. J. Nanoneurosci. 1.

Markin, V.S., and Albanesi, J.P. (2002). Membrane fusion: stalk model revisited. Biophys. J. 82, 693-712.

Pobbati, A. V., Stein, A., and Fasshauer, D. (2006). N- to c-terminal snare complex assembly promotes rapid membrane fusion. Science 313, 673-676.

Sollner, T., Whiteheart, S. W., Brunner, M., ErdjumentBromage, H., Geromanos, S., Tempst, P., and Rothman, J. E. (1993). Snap receptors implicated in vesicle targeting and fusion. Nature 362, 318-324.

Weninger, K., Bowen, M. E., Choi, U. B., Chu, S., and Brunger, A. T. (2008). Accessory proteins stabilize the acceptor complex for synaptobrevin, the 1:1 syntaxin/ snap-25 complex. Structure 16, 308-320.

Wiederhold, K., and Fasshauer, D. (2009). Is assembly of the snare complex enough to fuel membrane fusion? J. Biol. Chem. 284, 13143-13152.

Received: 28 May 2009; published online: 06 July 2009. Citation: Liu W and Parpura V (2009) Energetics of (dis)assembly of the ternary SNARE complex. Front. Neuroenerg. 1:5. doi: 10.3389/neuro.14.005.2009

Copyright: (c) 2009 Liu and Parpura. This is an openaccess publication subject to an exclusive license agreement between the authors and the Frontiers Research Foundation, which permits unrestricted use, distribution, and reproduction in any medium, provided the original authors and source are credited. 